\title{
Ramassage écologique des déchets plastiques
}

Khaled Moustafa

Manager of FrenXiv

Email: khaled.moustafa@frenxiv.org

La pollution environnementale est un problème global qui réduit la qualité de vie dans de nombreuses régions dans le monde. Certaines grandes villes sont étouffées par des quantités importantes de micro- et macro déchets organiques et inorganiques, tels que des métaux lourds, des gaz à effet de serre, des déchets alimentaires ${ }^{\stackrel{1}{ }}$, des déchets plastiques et des mégots de cigarette éparpillés ici et là. Ces derniers sont parmi les déchets les plus répandus sur terre. On les retrouve presque partout; dans les rues, les bords de routes, les terrasses des cafés, les gares routières, les plages, les parcs, etc. Chaque année, les municipalités dépensent des millions d'euros pour ramasser et traiter ces déchets.

De surcroit, les mégots de cigarette libèrent des produits toxiques - tels que le plomb et l'arsenic qui augmentent le risque de toxicité environnementale et d'empoisonnement des espèces locales $\stackrel{2}{ }$. Les déchets plastiques, en revanche, ne se dégradent pas facilement. Ils doivent être triés et recyclés en permanence pour réduire leur effet polluant. Cependant, le tri des déchets est un processus coûteux qui nécessite des moyens importants sur le plan financier et humain. Pour réduire ces coûts, je propose de dresser et d'utiliser des "chiens collecteurs" de déchets plastiques et des mégots de cigarettes pour en faciliter la collecte et le tri. Cela permettrait d'économiser une partie significative des ressources humaines et matérielles allouées au ramassage et à la séparation manuelle et mécanique de ces déchets.

Avec des millions de récepteurs olfactifs dans leurs nez, les chiens sont l'une des espèces les plus efficaces pour détecter et rechercher des objets variés qui dégagent des odeurs spécifiques. Lorsqu'ils sont dressés, les chiens peuvent guider des personnes non voyantes (chiens guides), détecter des substances toxiques, rechercher des truffes, localiser des mines explosives ou des drogues, retrouver des personnes disparues et même détecter certains types de cancers $\stackrel{3,4}{,}$, etc.

Avec de telles capacités, les chiens peuvent également être dressés pour ramasser des déchets plastiques et des mégots de cigarettes afin d'améliorer les efforts municipaux de la collecte et du tri écologiques de ces déchets. Les mégots de cigarettes et les objets en plastique ont souvent des odeurs particulières que les chiens peuvent sentir et, par conséquent, les ramasser efficacement. Des personnes mandatées et des points de collecte des déchets destinés à cet effet peuvent être installés dans des endroits précis d'une municipalité pour le bon déroulement du ramassage. 
Efficaces, faciles à dresser et à mettre en action, les chiens peuvent contribuer significativement à la gestion des déchets, réduisant ainsi les coûts du maintien des infrastructures publiques propres au mieux possible.

\section{Références:}

1 Moustafa, K. [Eating in public transportation: A behavior to avoid for health and sanitary purposes?]. Presse médicale 47, 606-610, doi:10.1016/j.Ipm.2018.05.015 (2018).

2 Moerman, J. W. \& Potts, G. E. Analysis of metals leached from smoked cigarette litter. Tobacco control 20 Suppl 1, i30-35, doi:10.1136/tc.2010.040196 (2011).

3 Guirao, A. et al. Trained dogs can identify malignant solitary pulmonary nodules in exhaled gas. Lung cancer 135, 230-233, doi:10.1016/j.lungcan.2019.06.008 (2019).

4 Taverna, G. et al. Olfactory system of highly trained dogs detects prostate cancer in urine samples. The Journal of urology 193, 1382-1387, doi:10.1016/j.juro.2014.09.099 (2015). 